\title{
Simulation of Electromagnetic Flow Meters Using the Boundary Element Method
}

\author{
Dr. René Friedrichs ${ }^{1}$, Dr. Eike Christian Weiß ${ }^{1}$ \\ ${ }^{1}$ SIKA Dr. Siebert \& Kühn GmbH \& Co. KG, Struthweg 7-9, 34260 Kaufungen (Germany), \\ friedrichs@sika.net
}

\begin{abstract}
:
This paper presents the simulation and optimization of the magnet system of an electromagnetic flow meter. The boundary element method (BEM) is used to solve the corresponding 3D magnetostatic Maxwell equations. A Fredholm integral equation of the second kind for the "microscopic" current on the surface of the paramagnetic pole shoes of the magnet system is derived. Discretizing the integral equation leads to a much smaller linear system as an adequate finite element formulation of the problem. For selected examples we show how the dramatically reduced computing time and effort for mesh preparation facilitates a fast and cost-efficient optimization of the magnet system of the electromagnetic flow meter.
\end{abstract}

Key words: electromagnetic flow meter, EMF, boundary element method, BEM, magnetostatic

\section{Magnetostatic Simulations Using Boundary Elements}

Recent years have seen a real increase in the use of numerical simulation in the design phase of the development of flow sensors. Thereby the finite element method (FEM) has established itself as analytical workhorse for structural mechanics, fluid dynamics and electrodynamics.

However, using FEM for magnetostatic simulations one encounters the problem that the magnetic fields are to be determined, in principle, at infinity. Therefore the simulation domain has to be truncated and assumptions on the symmetries of the fields at that boundary are to be made. This truncation of the domain and the meshing of the (hopefully) large enough domain is the most labour-intensive part in using FEM for magnetostatics. To circumvent this difficulty of domain based methods as FEM or FDM (finite difference method) we apply the boundary element method [1] to optimize the magnet system of an electromagnetic flow meter.

Exploiting the fact that the underlying equations describing the magnet system of the flow meter are assumed to be linear we derive in the following sections an integral equation for a "microscopic" or "virtual" surface current density. From this "microscopic" current density $\vec{j}$ on the surface of the paramagnetic materials of the magnet system the magnetic flux density within the flow tube of the magnetic flow meter can be calculated.

In this paper we restrict our investigations to a simple magnet system (see Fig. 1) with just one current carrying coil and one paramagnetic pole shoe to guide the magnetic field. However, our approach can easily be extended to more realistic situations with more then one source of the magnetizing field $\vec{H}(r)$, i.e. more coils, and different and complicated shaped magnetizable materials.

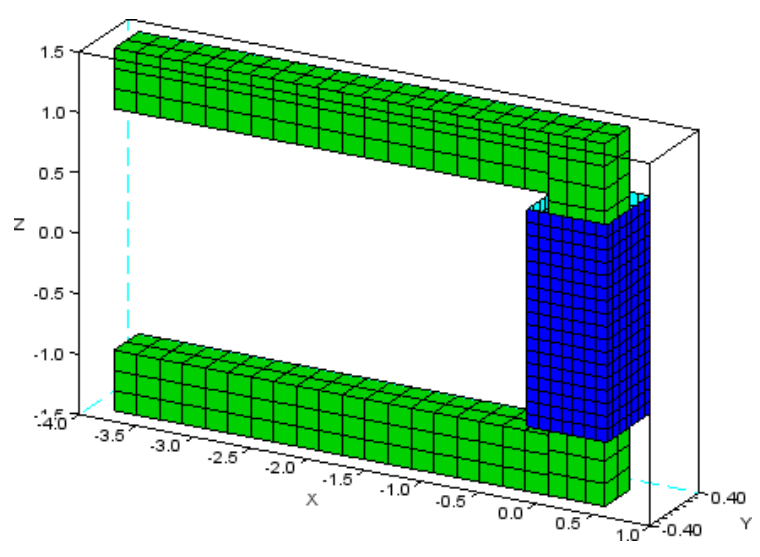

Fig. 1. Considered magnet system with current carrying coil (blue) and paramagnetic pole shoe (green). The flow is aligned in the $y$-direction and the flow tube (not shown) is positioned between the two arms of the pole shoe. 


\section{Basic Equations}

The basic equations describing the magnetic induction $\vec{B}(\vec{r})$ and the magnetizing field $\vec{H}(r)$ generated by macroscopic current $\vec{J}$ trough the coil and guided by the paramagnetic pole shoe with relative permeability $\mu_{r}$ are

$$
\begin{array}{r}
\nabla \cdot \vec{B}=0 \\
\text { and } \\
\nabla \times \vec{H}=\vec{J}
\end{array}
$$

together with the material equation

$$
\vec{B}(\vec{r})=\mu_{0} \mu_{r}(\vec{r}) \vec{H}(\vec{r}) .
$$

The assumption which facilitates our approach using the boundary element method is that the relative permeability $\mu_{r}$ is constant within the pole shoe. In particular $\mu_{r}$ is assumed to be independent of the magnetizing field $\vec{H}(\vec{r})$. The permeability everywhere outside the pole shoe is considered to be equal to one. Therewith we can conclude from equation (2) that the magnetic flux density is irrotational outside the current carrying coil except for the surface of the paramagnetic pole shoe. This motivates the following Biot-Savart law like ansatz for the magnetic flux density:

$$
\begin{gathered}
\vec{B}(\vec{r})=\frac{\mu_{0}}{4 \pi} \int_{V_{\text {Coil }}} d V^{\prime} \frac{\vec{J}\left(\vec{r}^{\prime}\right) \times\left(\vec{r}-\vec{r}^{\prime}\right)}{\left|\vec{r}-\vec{r}^{\prime}\right|^{3}}+ \\
+\frac{\mu_{0}}{4 \pi} \delta_{\partial V_{\text {PoleShoe }} d S^{\prime} \frac{\vec{j}\left(\vec{r}^{\prime}\right) \times\left(\vec{r}-\vec{r}^{\prime}\right)}{\left|\vec{r}-\vec{r}^{\prime}\right|^{3}} .} .
\end{gathered}
$$

From this equation we will be able to calculate the magnetic flux density at any desired position after the initially unknown "microscopic" current density $\vec{j}(\vec{r})$ is determined. Therefore we have to exploit that the tangential component of the magnetizing field $\vec{H}(\vec{r})$ is continuous at the surface of the pole shoe which results in the following discontinuity for magnetic flux density:

$$
\vec{n} \times\left(\frac{\vec{B}_{\text {inside }}}{\mu_{r}}-\vec{B}_{\text {outside }}\right)=\overrightarrow{0},
$$

whereby $\vec{n}$ denotes the normal vector pointing outside the volume of the pole shoe. Knowing that the surface current density $\vec{j}(\vec{r})$ results in a certain jump of the magnetic flux density which is given by

$$
\begin{gathered}
\vec{B}_{\text {in/out }}(\vec{r})=\vec{B}(\vec{r}) \pm \frac{\mu_{0}}{2} \vec{n} \times \vec{j}(\vec{r}) \\
\forall \vec{r} \in \partial V_{\text {PoleShoe }}
\end{gathered}
$$

we can derive a second relation between the magnetic flux density and the "microscopic" surface current density:

$$
\frac{-2}{\mu_{0}}\left(\frac{\mu_{r}-1}{\mu_{r}+1}\right) \vec{n}(\vec{r}) \times \vec{B}(\vec{r})=\vec{j}(\vec{r}) .
$$

Inserting equation (4) into equation (7) finally yields the desired equation for the unknown surface current

$$
\begin{gathered}
\vec{j}(\vec{r})=\vec{j}_{1}(\vec{r})+ \\
+\frac{\mu_{r}-1}{\mu_{r}+1} \vec{n}(\vec{r}) \times \oint_{\partial V_{\text {PoleShoe }}} d S^{\prime} \frac{\left(\vec{r}-\vec{r}^{\prime}\right)}{2 \pi\left|\vec{r}-\vec{r}^{\prime}\right|^{3}} \times \vec{j}\left(\vec{r}^{\prime}\right)(8)
\end{gathered}
$$

with

$$
\vec{j}_{1}(\vec{r})=\frac{\mu_{r}-1}{\mu_{r}+1} \vec{n}(\vec{r}) \times \int_{V_{\text {Coil }}} d V^{\prime} \frac{\left(\vec{r}-\vec{r}^{\prime}\right)}{2 \pi\left|\vec{r}-\vec{r}^{\prime}\right|^{3}} \times \vec{J}\left(\vec{r}^{\prime}\right) .
$$

\section{Numerical solution}

The above equation for the surface current density $\vec{j}(\vec{r})$ can easily be classified as a Fredholm integral equation of the second kind. Hence, after discretizing it, we compute its solution by using the method of successive substitutions [2]

$$
\begin{gathered}
\vec{j}_{n+1}(\vec{r})=\vec{j}_{1}(\vec{r})+ \\
+\frac{\mu_{r}-1}{\mu_{r}+1} \vec{n}(\vec{r}) \times \oint_{\partial V_{\text {PoleShoe }}} d S^{\prime} \frac{\left(\vec{r}-\vec{r}^{\prime}\right)}{2 \pi\left|\vec{r}-\vec{r}^{\prime}\right|^{3}} \times \vec{j}_{n}\left(\vec{r}^{\prime}\right)(9)
\end{gathered}
$$

Thereby we circumvent the use of a direct solver to solve the resulting linear system which is unfortunately not sparse.

\section{Example I: Permeability of the Pole Shoe}

As first example we have investigated how the permeability of the pole shoe influences the magnetic field within the flow tube. To get a sufficient spatial resolution we had to mesh the surface of the pole shoe with just 552 boundary elements resulting in only 1104 degrees of freedom. Exemplary, for a typical relative permeability of $\mu_{r}=1000$, the solution for the magnetic flux density within the flow tube and the meshing of the pole shoe is shown in Fig. 2 . 


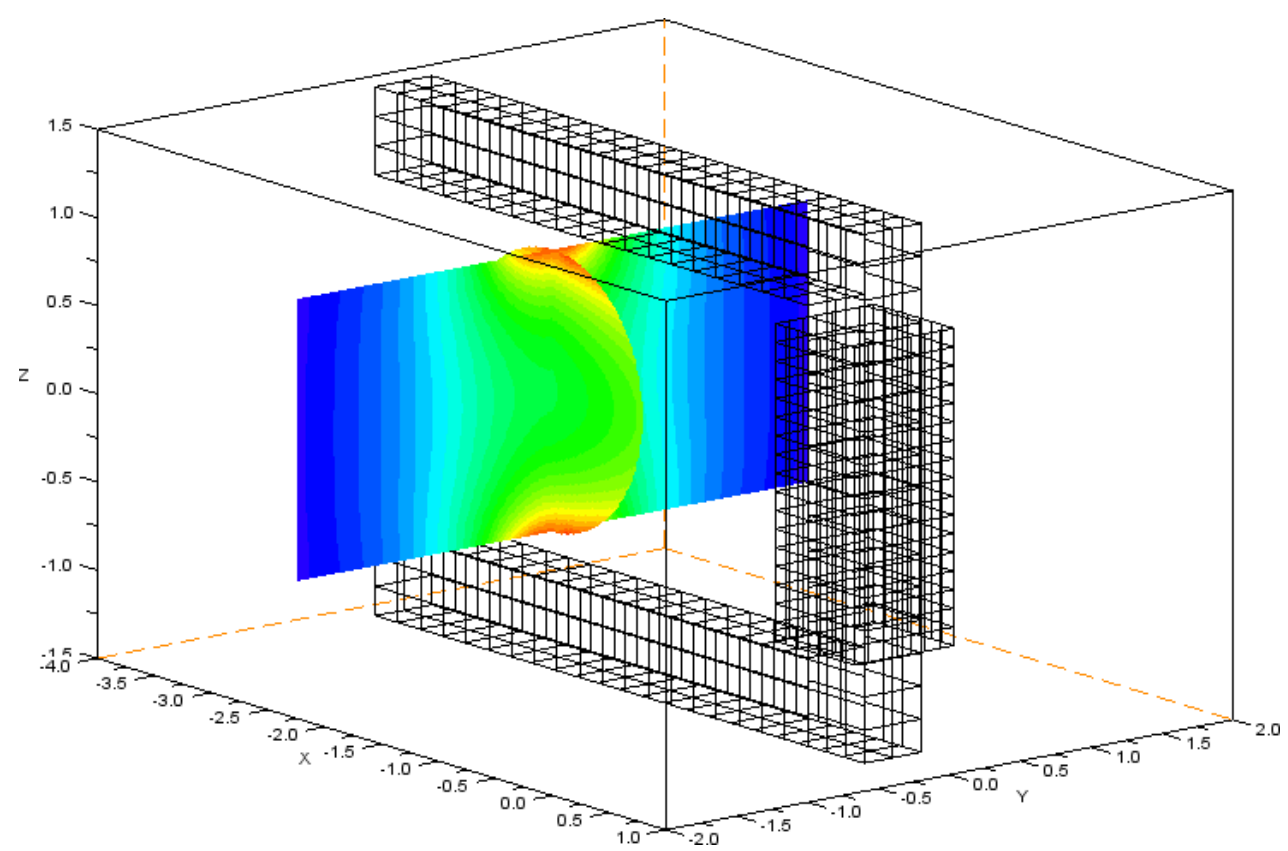

Fig. 2. Magnetic flux density within the circular flow tube aligned in the $y$-direction. The color indicates the magnitude of the B-Field (violet $=0 m T$, red $=24 m T$ ). The surface of the pole shoe was meshed with just 552 boundary elements. The simulation was done with just 1104 degrees of freedom.

To evaluate how good the magnet system guides the magnetic field we have computed the zcomponent of the flux density over the cross section of the flow tube (at the electrode positions).

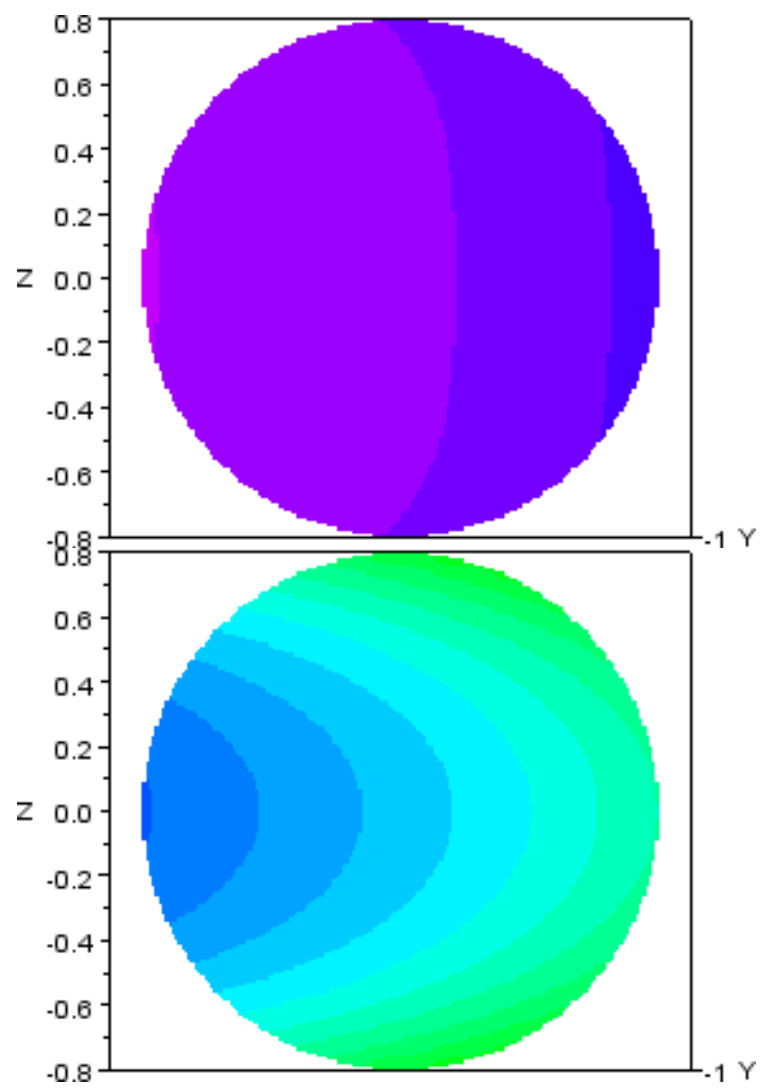

Fig. 3. Z-component of the flux density over the cross section of the flow tube (violet $=0 m T$, red $=24 m T)$. The electrodes are positioned at the left and right side of the cross section at $\mathrm{z}=0$.

Upper picture $\mu_{r}=10$. Lower picture $\mu_{r}=100$.

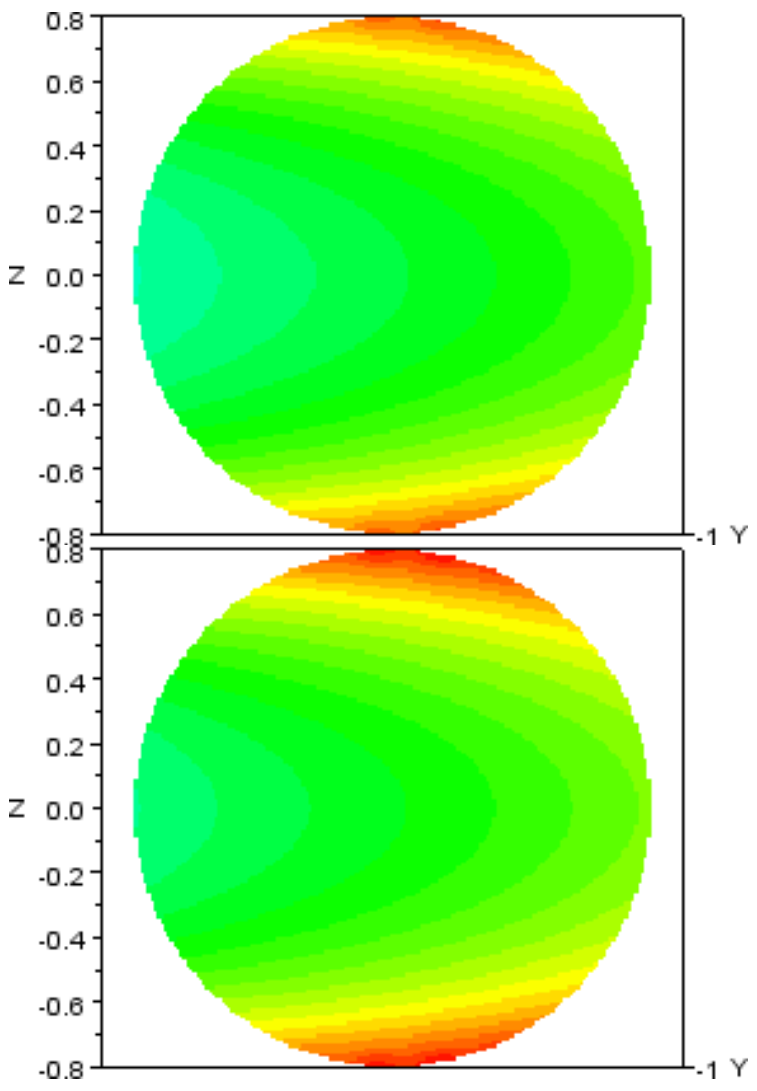

Fig. 4. Z-component of the flux density over the cross section of the flow tube (violet $=0 m T$, red $=24 m T)$. The electrodes are positioned at the left and right side of the cross section at $z=0$.

Upper picture $\mu_{r}=1000$. Lower picture $\mu_{r}=10000$. 
From Figs. 3 and 4 one can see that the magnetic field in the tube becomes stronger, and almost homogenous, at least in the region between both electrodes if the permeability of the pole shoe increases from 10 to 10000 .

To be able to judge if its worth to choose a material for the pole shoes with higher permeability we have computed the z- component of the magnetic field averaged over the cross section as function of the permeability (see Fig. 5). The resulting average can be used to estimate the sensitivity of the meter to flow, i.e. the measured voltage between the electrodes for a given flow-rate. The averaged flux density increases significantly only up to a relative permeability of 1000 .

\section{$B_{z}$ averaged over the cross section of the tube}

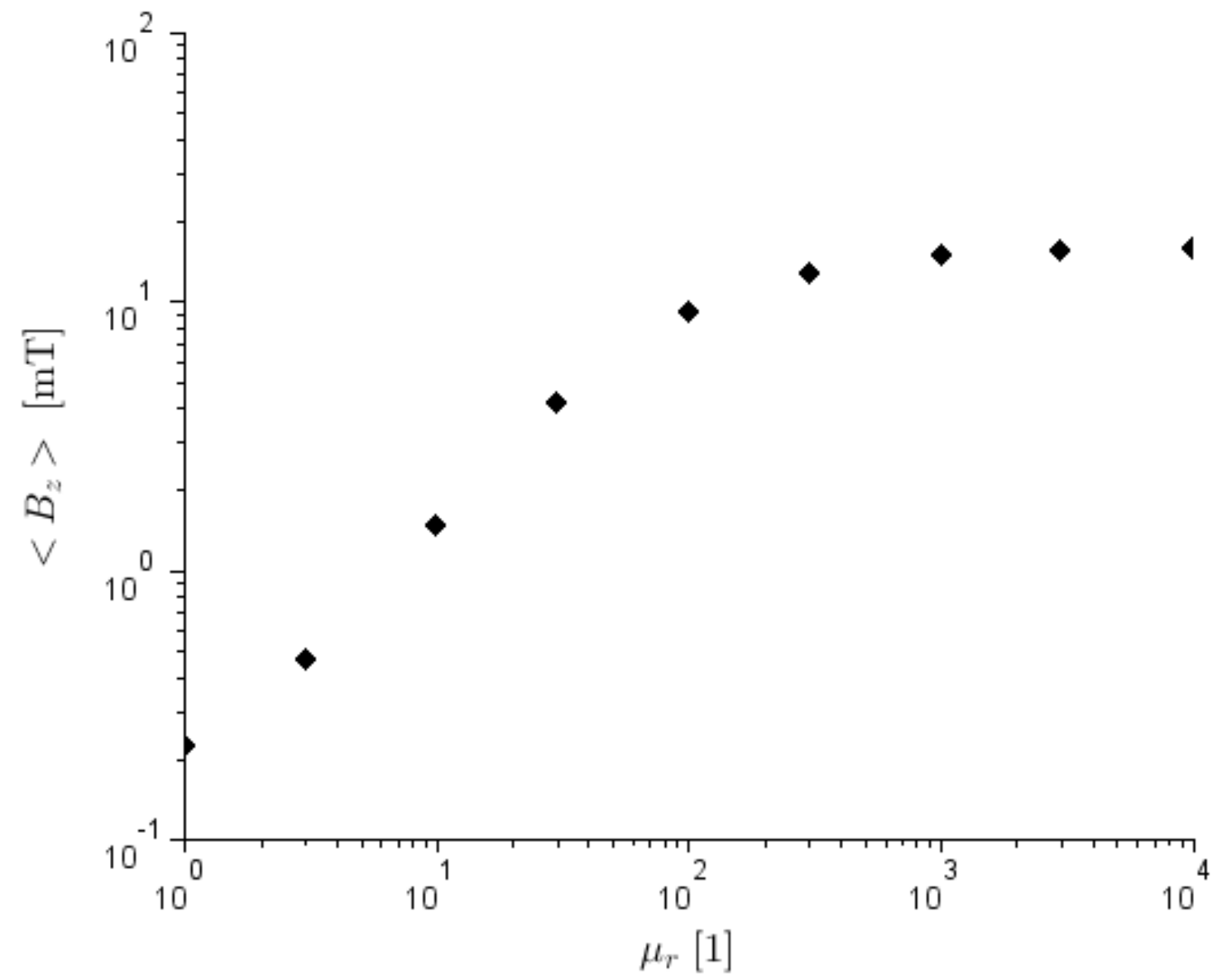

Fig. 5. Z-component of the magnetic flux density averaged over the cross section. The averaged flux density increases significantly only up to a relative permeability of 1000. 


\section{Example II: Tilting the Coil}

As second example we have investigated how a tilting of the current carrying coil with respect to the flow tube and the pole shoe influences the magnetic field within the flow tube. This modification of the geometry does not require a new meshing. This is in contrast to the finite element method (FEM) and an essential advantage of the boundary element method (BEM).

Exemplary, for the relative permeability of $\mu_{r}=1000$ and a tilt angle of $5^{\circ}$ the solution for the magnetic flux density within the flow tube is shown in Fig. 6. For this permeability the tilting of the coil has a negligible effect on the magnetic field within the flow tube.

For a tilt angle of $5^{\circ}$ a noticeable effect can be observed only for an untypical small permeability of the pole shoe of $\mu_{r}=10$ as shown in Fig. 7.

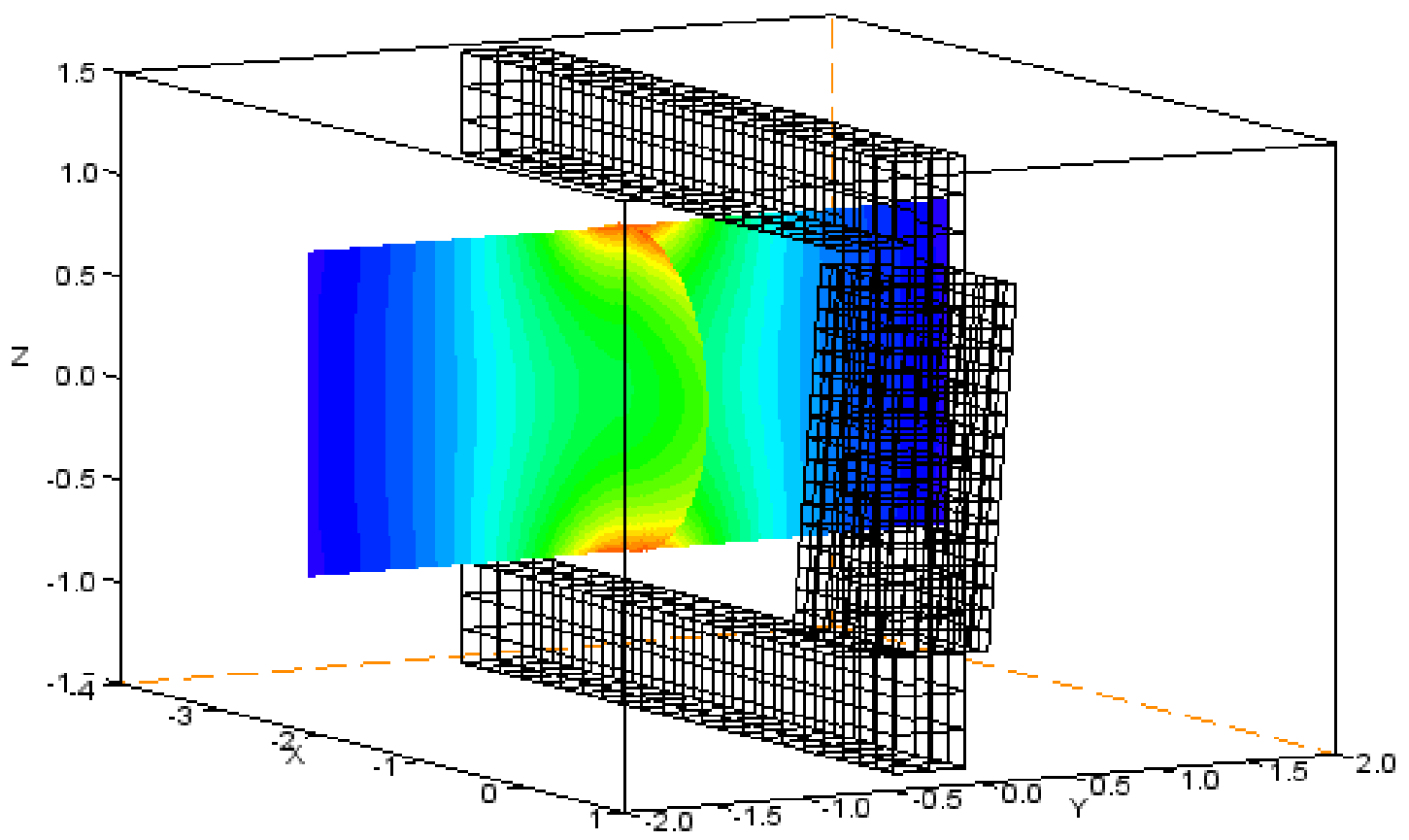

Fig. 6. Magnetic flux density within the circular flow tube aligned in the $y$-direction for a slightly tilted coil (5). The relative permeability of the pole shoe was 1000. The color indicates the magnitude of the B-Field (violet $=$ $O m T$, red $=24 m T$ ).

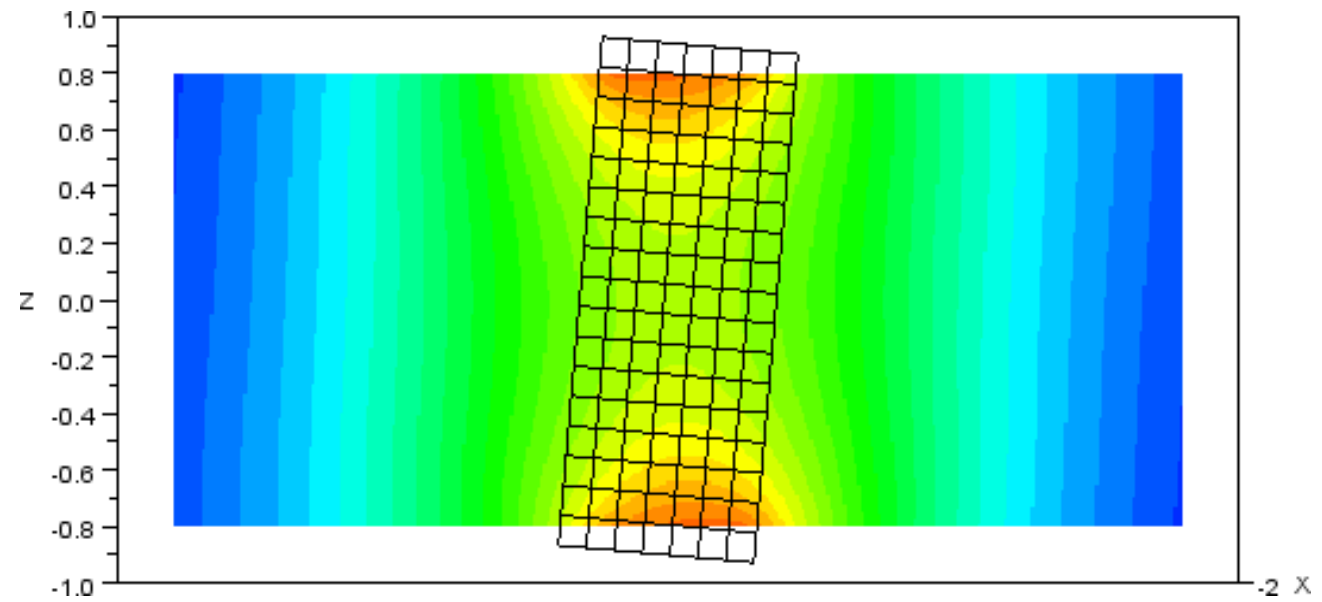

Fig. 7 Magnetic flux density within the flow tube for a slightly tilted coil (59. The relative permea bility of the pole shoe was only 10. The color indicates the magnitude of the B-Field (violet $=0 m T$, red $=1.1 \mathrm{mT}$ ). 


\section{Summary and Conclusion}

We have shown on two examples how the magnetic field generated by a magnetic system of an electromagnetic flow meter can be computed using the boundary element method (BEM) The BEM has two essential advantages:

Only the surfaces of the magnetizable parts of the magnet system must be meshed. This leads to a much reduced meshing effort. If just positions and no shapes of the parts of the magnet system are changed even no new meshing is required.

The primary quantity, which is solved for, is the "microscopic" surface current density which is unknown only on the surface of the magnetizable parts. This leads to a dramatically reduced number of degrees of freedom. Even simulations with 1000 degrees of freedom yield solutions for the magnetic field within the flow tube of the flow meter with sufficient accuracy and smoothness, i.e. differentiability, for optimization. For all shown examples, using just a common desktop computer, it took less then 2 minutes to compute the magnetic field within the flow tube. Hence for more realistic, i.e. more complicated magnet systems, with about 5000 degrees of freedom we expect the simulation time to be considerably smaller then one hour.

So the boundary element method (BEM) facilitates a fast and cost-efficient optimization of the magnet system of electromagnetic flow meters.

\section{References}

[1] A. H.-D. Cheng, D. T. Cheng, Engineering Analysis with Boundary Elements 29, 268-302 (2005); doi:10.1016/j.enganabound.2004.12.001

[2] C. Pozrikidis, Boundary Integral and Singularity Methods for Linearized Viscous Flow (1992) 